\title{
THE COHOMOLOGY OF PRINCIPAL BUNDLES, HOMOGENEOUS SPACES, AND TWO-STAGE POSTNIKOV SYSTEMS
}

\author{
BY J. P. MAY ${ }^{1}$
}

Communicated by F. P. Peterson, November 13, 1967

In this note, we state some results on the cohomology of topological spaces that have been obtained by a study of the Eilenberg-Moore spectral sequence. Details and proofs will appear in [11].

We consider either of the essentially equivalent diagrams:

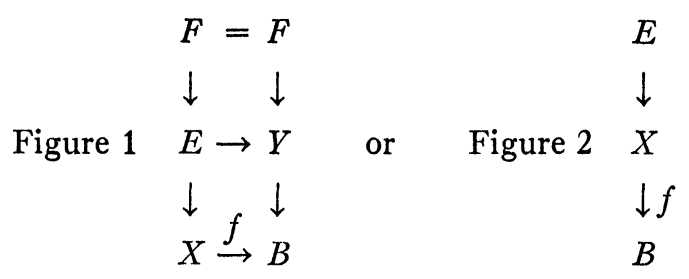

In Figure 1, $Y \rightarrow B$ is an acyclic fibration with fibre $F$ and $E \rightarrow X$ is the fibration induced by $f: X \rightarrow B$. In Figure $2, f: X \rightarrow B$ is a Serre fibration with fibre $E$. We assume (in both cases) that $B$ is pathwise connected and simply connected. Our results concern the cohomology of $E$.

Let $\Lambda$ be a commutative Noetherian ring. Cohomology will be taken with coefficients in $\Lambda$ except where explicitly stated otherwise. We assume that $H^{*}(B)$ is $\Lambda$-flat and that $H^{*}(X)$ and $H^{*}(B)$ are of finite type as $\Lambda$-modules. Then there is a spectral sequence of differential $\Lambda$-algebras $\left\{E_{r}\right\}$, defined by Eilenberg and Moore [6], which satisfies the conditions:

(i) $E_{2}=\operatorname{Tor}_{H^{*}(B)}\left(\Lambda, H^{*}(X)\right)$, where $H^{*}(X)$ has the structure of left $H^{*}(B)$-module determined by the map $f^{*}: H^{*}(B) \rightarrow H^{*}(X)$, and

(ii) $\left\{E_{r}\right\}$ converges to $H^{*}(E)$, in the sense that $E_{\infty}$ is isomorphic to the associated graded algebra $E^{0} H^{*}(E)$ of $H^{*}(E)$ with respect to a suitable filtration.

With these hypotheses and notations, we have the following result.

TheOREM. Let $H^{*}(B)$ be a polynomial algebra, and let $X$ be one of the following:

(a) $X=B G$, where $G$ is a compact connected Lie group such that $H^{*}(B G)$ is a polynomial algebra on even degree generators.

${ }^{1}$ Research partially supported by N.S.F. grant number GP-5609. 
(b) $X=B G$, where $G$ is $\left(Z_{2}\right)^{\text {a }}$, or $0(n)$, or a compact connected Lie group such that $H^{*}\left(B G ; Z_{2}\right)$ is a polynomial algebra on $n$ generators, where $G$ contains a subgroup isomorphic to $\left(Z_{2}\right)^{n}=Z_{2} \oplus \cdots \oplus Z_{2}$.

(c) $X=X_{i} K\left(\pi_{i}, n_{i}\right)$, where each $n_{i} \geqq 2$ and each $\pi_{i}$ is a finitely generated Abelian group subject only to the proviso that if $n_{i}=2$, then $\pi_{i}$ has no cyclic summands of the form $Z_{2} h, h>1$.

Then, in case (a), $E_{2}=E_{\infty}$ and therefore $E^{0} H^{*}(E)$ is isomorphic to $\operatorname{Tor}_{H^{*}(B)}\left(\Lambda, H^{*}(X)\right)$. In cases (b) and (c), assume further that $\Lambda=Z_{2}$. Then $E_{\infty}$ is effectively computable, up to any specified finite total degree, from knowledge of $f^{*}: H^{*}\left(B ; Z_{2}\right) \rightarrow H^{*}\left(X ; Z_{2}\right)$.

The proofs in cases (b) and (c) will actually yield an algorithm for the computation of $E^{0} H^{*}\left(E ; Z_{2}\right)$ in terms of $f^{*}$; and we shall also obtain conditions on $f^{*}$ sufficient to guarantee that $E_{2}=E_{\infty}$.

The hypotheses on $G$ in (a) are satisfied when $\Lambda$ is a field of characteristic $p$ and $G$ has no $p$-torsion or when $\Lambda$ is arbitrary and $G$ has no torsion. The only conspicuous missing general case occurs when $\Lambda=Z_{2}, H^{*}\left(B G ; Z_{2}\right)$ is a polynomial algebra, but $G$ does have 2 -torsion. The result in case (b) largely fills this gap, since the hypotheses on $G$ in (b) are satisfied, for example, when $G$ is $\mathrm{SO}(n), G_{2}$, or $F_{4}$.

Before describing the key elements in the proof of the theorem, we single out some of its most important applications. First, taking Figure 1 to be a classifying diagram, we obtain the following result.

CoROLLARY. Let $U$ be a connected topological group such that $H^{*}(B U)$ is a polynomial algebra and let $G$ be one of the Lie groups specified in (a) or (b) of the theorem. Let $E \rightarrow B G$ be a principal bundle, with group $U$, classified by a map $f: B G \rightarrow B U$. Then, in case (a), $E^{0} H^{*}(E)$ is isomorphic to $\operatorname{Tor}_{H^{*}(B U)}\left(\Lambda, H^{*}(B G)\right)$. In case (b), $E^{0} H^{*}\left(E_{;} Z_{2}\right)$ is effectively computable, up to any specified finite total degree, from knowledge of $f^{*}: H^{*}\left(B U ; Z_{2}\right) \rightarrow H^{*}\left(B G ; Z_{2}\right)$.

Similarly, if $G$ is a closed subgroup of $U$, we can take Figure 2 to be the fibration $U / G \rightarrow B G \stackrel{f}{\rightarrow} B U$, where $f$ is induced by the inclusion $G \rightarrow U$. We then obtain the following result.

COROLLARY. Let $U$ be a connected topological group such that $H^{*}(B U)$ is a polynomial algebra. Let $G$ be a closed subgroup of $U$, and suppose that $G$ is isomorphic to one of the groups specified in (a) or (b) of the theorem. Then in case (a), $E^{0} H^{*}(U / G)$ is isomorphic to $\operatorname{Tor}_{H^{*}(B U)}\left(\Lambda, H^{*}(B G)\right)$. In case (b), $E^{0} H^{*}\left(U / G ; Z_{2}\right)$ is effectively computable (up to any finite total degree) from knowledge of $f^{*}: H^{*}\left(B U ; Z_{2}\right)$ $\rightarrow H^{*}\left(B G ; Z_{2}\right)$. 
These corollaries are of course of greatest interest when $U$ is a compact Lie group, but it should be observed that $U$ could also be, for example, the infinite unitary, symplectic, or special orthogonal group.

Case (a) of the second corollary is a generalization of a conjecture of P. Baum [1] and extends results of Borel [3] for the case when $U$ is a compact Lie group and rank $G=$ rank $U$. Borel's result will be used in the proof of case (a) of our theorem. By a theorem of Cartan [5], which has been reproven and generalized by Baum and Smith [2], $H^{*}(U / G)$ and $\operatorname{Tor}_{H^{*}(B U)}\left(\Lambda, H^{*}(B G)\right)$ are isomorphic as algebras when $\Lambda$ is the real numbers and $U$ is a compact Lie group. It seems possible that this strengthened form of the corollary is true when $\Lambda$ is any field of characteristic unequal to two, but the author has made no attempt to prove this. It follows from a universal coefficient theorem argument that if $\Lambda=Z$, then $H^{*}(U / G)$ and $\operatorname{Tor}_{H^{*}(B U)}\left(\Lambda, H^{*}(B G)\right)$ are isomorphic as Abelian groups. Further discussion of case (a) of this corollary and examples giving explicit computations may be found in Baum's paper [1].

Case (b) of the second corollary extends results obtained by Borel in [4]. In that paper, he developed an analogy between the roles played by maximal tori in real cohomology and maximal 2-groups $\left(Z_{2}\right)^{n}$ in mod 2 cohomology. There is a simple consequence of the Cohen-Macauley theorem, as generalized by Auslander and Buchsbaum, which helps to explain this analogy and can be used to reprove many of Borel's results. By use of this result and an argument due to Baum, the general cases of parts (a) and (b) of our theorem can be reduced to the special cases when $G$ is a torus $T^{q}$ and when $G$ is a 2group $\left(Z_{2}\right)^{q}$. Since $B T^{q}=K\left((Z)^{q}, 2\right)$ and $B\left(Z_{2}\right)^{q}=K\left(\left(Z_{2}\right)^{q}, 1\right)$, all parts of the theorem deal primarily with the case when $X$ is a product of $K(\pi, n)$ 's.

Case (c) of the theorem is of greatest interest when $B=X_{i} K\left(\varnothing_{j}, m_{j}\right)$. Here, with $F=\Omega B$, Figure 1 defines a two-stage space $E$ and, as shown by Adams, such spaces are universal examples for secondary cohomology operations. Our results show how to compute the mod 2 cohomology of such a space from knowledge of $f^{*}: H^{*}\left(B ; Z_{2}\right) \rightarrow H^{*}\left(X ; Z_{2}\right)$, provided that $B$ is simply connected and that $X$ satisfies the hypotheses in (c). (Part (b) of the theorem covers the additional case $X=K\left(\left(Z_{2}\right)^{q}, 1\right)$, and our detailed results will cover, less satisfactorily, the case $X=X_{i} K\left(\pi_{i}, n_{i}\right)$, where it is only assumed that each $n_{i} \geqq 1$ and that $\pi_{i}$ has no cyclic summands $Z_{2} h, h>1$, if $n_{i}=1$.) If $X=K(\pi, n)$ and $B=K\left(\pi^{\prime}, n^{\prime}\right), n^{\prime}>n+1$, then Figure 1 defines a two-stage Postnikov system, and our results therefore solve, in a sense, the problem of computing the mod 2 cohomology of (almost) any space having 
only two nonvanishing homotopy groups. Our solution is incomplete in that no procedure is given for determining $H^{*}\left(E ; Z_{2}\right)$ as an algebra from its associated graded algebra and that no procedure is given for determining the Steenrod operations. Of course, one would ideally like a characterization of $H^{*}\left(E ; Z_{2}\right)$ in terms of generators and relations, but such a result seems to be beyond the reach of our methods, or of any other methods presently known.

Under appropriate stability assumptions on $f: X \rightarrow B$, Kristenson [8], Massey and Peterson [9], and Smith [12] have computed $H^{*}\left(E ; Z_{2}\right)$ as an algebra, where $E$ is a two-stage space. Their results also give partial information on the Steenrod operations. The only previous work known to the author in the unstable case is that of Hirsch, Cockcroft, and Barcus; their results essentially give methods for computing $H^{*}(E)$ additively by means of a complex involving $H^{*}(\Omega B)$ and $C^{*}(X)$. In [7], Hirsch gives a conjecture which, if true, would give a procedure for the additive computation of $H^{*}\left(E ; Z_{2}\right)$ in terms of $H^{*}\left(\Omega B ; Z_{2}\right)$ and $H^{*}\left(X ; Z_{2}\right)$. His conjecture is easily seen to be equivalent to the statement that $E_{2}=E_{\infty}$ in the EilenbergMoore spectral sequence, and our detailed results will therefore give a sufficient condition for its truth. Smith's notion of stability is that $f^{*}: H^{*}\left(B ; Z_{2}\right) \rightarrow H^{*}\left(X ; Z_{2}\right)$ be a morphism of Hopf algebras, and he shows that $E_{2}$ is also equal to $E_{\infty}$ in this case. Nevertheless, it seems most unlikely to the author that Hirsch's conjecture is true in general.

The proof of our theorem is entirely algebraic. The crucial point is a complete description, in terms of certain cohomology operations, of all higher differentials in the Eilenberg-Moore spectral sequence. This algebraic work goes through in much greater generality than is needed for the present applications and has other interesting consequences, some of which will be mentioned below. Thus let $U$ be a DGA-algebra over $\Lambda$ and let $M$ be a right and $N$ a left differential graded $U$-module. Under mild technical hypotheses, there is a spectral sequence $\left\{E_{r}(M, U, N)\right\}$ such that $E_{2}=\operatorname{Tor}_{H(U)}(H(M), H(N))$ and $\left\{E_{r}\right\}$ converges to TorU $(M, N)$. In this spectral sequence, every higher differential is determined by a specific matric Massey product. These operations, which will be defined in [10], generalize the ordinary Massey products in that they are defined on appropriate $n$ tuples of matrices rather than just on $n$-tuples of elements.

The spectral sequence studied in our theorem is $\left\{E_{r}\left(\Lambda, C^{*}(B)\right.\right.$, $\left.\left.C^{*}(X)\right)\right\}$. To prove the theorem, we first use the fact that $H^{*}(B)$ is a polynomial algebra to show that the only cochains of $B$ that enter into the computation of the relevant matric Massey products are iterated $U_{1}$-products of representative cocycles for elements of a set 
of generators for $H^{*}(B)$. Using the previously indicated fact that $X$ may be assumed to be a product of $K(\pi, n)$ 's, we next construct a homology isomorphism of DGA-algebras $\alpha: C^{*}(X) \rightarrow H^{*}(X)$. We may then replace $\left\{E_{r}\left(\Lambda, C^{*}(B), C^{*}(X)\right)\right\}$ by $\left\{E_{r}\left(\Lambda, C^{*}(B), H^{*}(X)\right)\right\}$. By keeping track of how $\alpha$ acts on iterated $U_{1}$-products, we can show how to calculate, without recourse to the cochain level, all matric Massey products relevant to the computation of the higher differentials. This yields an algorithm for the computation of $E_{\infty}\left(\Lambda, C^{*}(B), H^{*}(X)\right)$ in terms of $f^{*}: H^{*}(B) \rightarrow H^{*}(X)$. When $X=K\left(\left(Z^{q}\right), 2\right)=B T^{a}, \alpha$ annihilates all $U_{1}$-products. This implies that all relevant matric Massey products are zero, hence that the spectral sequence collapses, in case (a). In cases (b) and (c), sufficient conditions on $f^{*}$ can be found for $\alpha$ to annihilate those $U_{1}$-products (the images under $f^{*}: C^{*}(B) \rightarrow C^{*}(X)$ of the cochains of $B$ described above) which enter into the computation of the higher differentials, and thus sufficient conditions for the spectral sequence to collapse can be obtained.

With appropriate choices of chains or cochains for $M, U$, and $N$, the description of the higher differentials in $\left\{E_{r}(M, U, N)\right\}$ in terms of matric Massey products has other, more immediate, applications to algebraic topology. In particular, it leads (under mild technical hypotheses) to complete descriptions of the kernels of the following maps in terms of matric Massey products:

(1) The homology suspension $\sigma_{*}: H_{*}(G) \rightarrow H_{*}(B G)$, where $G$ is a topological monoid (associative $H$-space with unit).

(2) $p_{*}: H_{*}(Y) \rightarrow H_{*}(B)$, where $p: Y \rightarrow B$ is a principal fibration with fibre a topological monoid $G$.

(3) $i_{*}: H_{*}(X) \rightarrow H_{*}\left(X \times{ }_{G} E G\right)$, where $G$ is a topological group (with universal bundle $E G \rightarrow B G$ ) which operates on $X$.

(4) The cohomology suspension $\sigma^{*}: H^{*}(B) \rightarrow H^{*}(\Omega B)$.

(5) $i^{*}: H^{*}(Y) \rightarrow H^{*}(F)$, where $F \stackrel{i}{\rightarrow} Y \rightarrow B$ is a Serre fibration.

(6) $q^{*}: H^{*}(X) \rightarrow H^{*}(E)$, where $q: E \rightarrow X$ is a Serre fibration induced from an acyclic fibration $Y \rightarrow B$.

These results suggest that matric Massey products have a fundamental role to play in algebraic topology. A detailed study of their properties will appear in [10].

\section{BIBLIOGRAPHY}

1. P. Baum, On the cohomology of homogeneous spaces (to appear).

2. P. Baum and L. Smith, The real cohomology of differentiable fibre bundles (to appear).

3. A. Borel, Sur la cohomologie des espaces fibres principaux et des espaces homogènes de groupes de Lie compacts, Ann. of Math. 57 (1953), 115-207. 
4. - La cohomologie mod 2 de certains espaces homogenes, Comm. Math. Helv. 27 (1953), 165-197.

5. H. Cartan, La transgression dans un groupe de Lie et dans un espace fibre princi pal, Colloque de topologie, Bruxelles (1950), 57-71.

6. S. Eilenberg and J. C. Moore, Homological algebra and fibrations, Colloques de topologie, Bruxelles (1964), 81-90.

7. G. Hirsch, Cohomologie d'un espace de Postnikov (cas non stable) (to appear).

8. L. Kristenson, On the cohomology of spaces with two non-vanishing homotopy groups, Math. Scand. 12 (1963), 83-105.

9. W. S. Massey and F. P. Peterson, The mod 2 cohomology structure of certain fibre spaces, Mem. Amer. Math. Soc. 74 (1967).

10. J. P. May, Matric Massey products (to appear).

11. - The structure and applications of the Eilenberg-Moore spectral sequences (to appear).

12. L. Smith, The cohomology of stable tro-stage Postnikov systems, Illinois J. Math. (to appear).

University of Chicago 